\title{
Vibro-Tactile Interface for Enhancing Piloting Abilities during Long Term Flight
}

\author{
Sylvain Cardin, Frédéric Vexo, Daniel Thalmann \\ Virtual Reality Laboratory, Ecole Polytechnique Fédérale de Lausanne (EPFL), \\ E-mail:\{sylvain.cardin; frederic.vexo; daniel.thalmann\} @epfl.ch
}

\begin{abstract}
Heading perception of an aircraft becomes uneasy under disturbing spatial disorientation for a single pilot performing the daily tasks inherent to a long term flight. Sleepiness, movements and other activities introduced by vital function necessity reduce the attention and the awareness of the current aircraft situation. This paper presents the development of a system which aims at decreasing the attention needs for maintaining an aircraft's attitude and take corrective action when the autopilot goes off bound. An embedded system has been integrated in the pilot's clothing. It sends vibro-tactile feedback to the pilot when his aircraft becomes off balance. The system also dynamically localizes the position of the actuators in order to insure a feedback constant in space independently form the pilot posture and movements. A series of tests have been conducted to validate the interest of this localization by showing a slight improvement in the response time needed to take corrective action. By increasing the pilot's own feeling about his plane's orientation, the system provides a complementary tool to improve exhausting long flight conditions.
\end{abstract}

\section{Introduction}

Long term flights are an exhausting task for pilots and crew members alike. Regular military operations can require a plane flying for an unlimited amount of time by refueling the plane regularly. A single pilot can be sent on a $20 \mathrm{~h}$ flight mission at any time of the day or night. One other famous example is the first nonstop unrefueled airplane flight around the world completed in 1986 by Dick Rutan and Jeana Yeager. They flew for more than 9 days aboard the Voyager, a trimaran airplane, to cover the $40203 \mathrm{~km}$ to return to their starting point. While performing such extreme record, a single person could only rely on the auto pilot to alert him from a specific danger. This sometimes leads to dramatic accidents due to loose of attention from sleep deprivation [i]. Sleep deprivation increases response time and can induce misjudgments of situations.

Human condition is a crucial part for the success of such missions. The pilot has to fly the aircraft during days and nights allowing him minimal loss of attention and sleeping time. Movement and posture variation, like picking up an object from a compartment, feeding time, or even relaxation and napping, reduce the pilot's attention and mask some changes in aircraft behavior. In accordance with the sensitivity of the aircraft commands, a small variation of the balance can lead to a dramatic change in the aircraft's trajectory. The balance perception is, in every day life, done via visual, vestibular and proprioceptive cues. Experiments confirm also that the head axis spatial reference plays an important role in orientation perception [ii]. Stress, induced by variation of $g$-forces inherent to airplane and boat traveling, fools the vestibular and proprioceptive cues. This phenomena lead to well known motion sickness. Spatial disorientation is almost total if we add to this stress a limited visibility due to weather condition, as night or fog. Also disorientation and stress, while being waked up by alarm signals, decrease dramatically the time to regain control of the aircraft. Disorientation is an example of a major cause in helicopter crashes according to the United States military [iii].

The aim of this paper is to present the current development of a wearable vibrating system to help the pilot to sustain the plane's balance with minimal attention. This system will alert when the autopilot becomes out of the target range and inform the pilot instantaneously of the plane's attitude. We will present first the related work on device for navigation enhancement and vibrating system development. Then we will explain our system architecture and the technical aspects of our development. The next part will develop the functioning principles of such self localized vibrating systems. A series of tests and validation procedures followed by their results and further discussion will conclude this paper. 


\section{Vibro-tactile Navigation Enhancement}

Many researches are going on the use of vibrating system to enhance navigation, especially when visual feedback is reduced, absent or already overloaded. Vibrating devices have been used in several applications to enhance navigation ability. Most of those applications aim at giving spatial orientation information to the user through vibrating feedback. One example of this application is the research done on vibrating belts for navigation way point enhancement [iv]. The device consists in a belt containing 8 vibrators which vibrates according to the position of the target given by GPS coordinates. The system has been tested on board of a helicopter and a boat to detect its influences on the trajectory taken by pilot by using direct GPS coordinate and/or tactile feedback. Prototype of tactile display onto the user body have been tested on aircraft crew members by the Naval Aerospace Medical Research Laboratory for enhancing spatial orientation and situation awareness in military applications[v]. Validation and tests results show the improvement in terms of performances and reducing workloads of the crew members of the $18^{\text {th }}$ flight test of the US Air Force. The device composed by only a few pneumatic and vibrating actuators was test on combat plane simulators and on field helicopter flights. These case studies definitely show the interest and usability of a tactile interface for crew member assistance.

Knowing the interest of the user community is one part, but vibrotactile rendering of spatial information leads to some questions of ergonomic and cognitive perception. Research from the TNO Human Factors Organization lead by Professor Jan B.F. van Erp on vibrotactile interfaces provide useful guidelines for future application development [vi]. This paper presents guidelines on tactile information coding, their usability, threshold and pitfalls in terms of subjective magnitude, frequency, location and temporal patterns.

Since the early sixties, researches have been conducted on the sensitivity of the human body to vibrotactile stimulation in term of amplitude, frequency and spatial cues [vii][viii]. Based on those studies, the main idea is that subjective magnitude [ix] and frequency allows up to 9 discernable levels to code the information $[\mathrm{x}]$. The temporal sensitivity of the skin is very high with a detection threshold down to $10 \mathrm{~ms}$ [xi]. Spatial accuracy of the human skin is variable according to the area considered while the finger end can discern stimuli separated by only a couple of millimeters while that can raise up to 3 centimeters[xii]. It leads us to build a vibro tactile device which modulates the magnitude and frequency of the vibration input onto a large surface of the user body.

Although many pitfalls still have to be taken in account in the design process. Pattern recognition, for example, can suffer from spatial masking. This phenomenon occurs when close stimuli overlap in time. The apparent location of a stimulus can be induced by the presence of two distinct stimuli around this location [xiii]. Temporal effects occurs when two stimuli appears closely in time on the same location. The sensation provoked by the second stimuli can be drastically modified if the times step between then is less than a few hundred milliseconds [xiv]. Modulating the frequency of the stimuli can be done to reduce the influence of such masking. Continuous excitation of the skin by vibration leads in overwhelming the skin sensors and attenuate the perception of the phenomena [xv].A solution consist in using burst stimuli of $200 \mathrm{~ms}$ on for $800 \mathrm{~ms}$ off as during the experience done at the university of Oxford [xvi]. However it shows that even under those conditions, spatiotemporal masking is still a major limitation in pattern recognition. To prevent such a pitfall, only one type of information will be passed to the user through the vibro-tactile device and the evolution of the stimuli will be continuous in space. In order to achieve this continuity, spatial information about the actuators has to be taken in account.

Implementation and testing of a vibrotactile vest for the torso have been developed at the University of Pohang in Korea [xvii]. Some interesting tests have been realized on how to render various shapes in space in correlation to the static position of the vibrators. The results show that users feel the presence of moving 3D object in the vibration space and are able to mentally reconstruct spatial information and orientation of those objects. The movement is an important vector of information for those types of interfaces since it allows mental reconstruction according to our self position perception [xviii]. Speaking about motion, a vibrating device has been tested for the purpose of reducing spatial disorientation among astronauts during weightlessness [xix]. The device gives the position of a "virtual" gravity by rendering a constant direction in space representing the bottom direction. The major interest in this paper consists in using the user movement as the variable of the system.

According to the literature, vibro-tactile feedback seems an intuitive way to render orientation information. It could decrease the attention needs traditionally required to check on board instruments while stimulating the user to reduce the effects of sleepiness. Taking in account the user positioning allows spatial consistency of the represented feedback for better mental reconstruction of multidimensional information. The working principles of our application are based on those paradigms. 


\section{Vibrotactile interface}

Our efforts have been focused on the development of a vibrotactile device which alerts the pilot of an aircraft about its attitude. This system aims at providing intuitive information for taking corrective action when the autopilot goes off bound. This problem appears when the external conditions influence the attitude more than could be corrected automatically. The system renders through the skin of the pilot, the plane attitude characterized by the pitch and roll angles. The system will recover this data to generate a vibrating feedback according to the current situation.

Four different vibrating patterns have been used and described below illustrated by a schematic and screenshot of the flight simulator. For each of them a symbolic schematic shows the general principle. The circles represent the actuators state. Red color symbolizes a high stimulation while a grey symbolizes a neutral state. Positions of the actuators on the schema have been idealized for symbolic purposes. In the two last modes a screenshot of the graphical interface have been added to show the state and position of the actuators according to the attitude of the plane symbolized by the green square.

Our first question is how to render such spatial information onto the pilot's skin. The simplest paradigm that could be implemented is to use 4 actuators located on the upper chest. The front and the back of the torso are dedicated to the pitch and the left and right shoulders to the roll. In this configuration, the system will send vibration to the left when the plane leans left. The vibrating intensity is directly proportional to the sine of the pitch and roll angles, without taking in account any information about the pilot position. The figures below present on the right the current situation of the airplane as it runs on the simulator and on the left the graphical interface of the feedback engine.
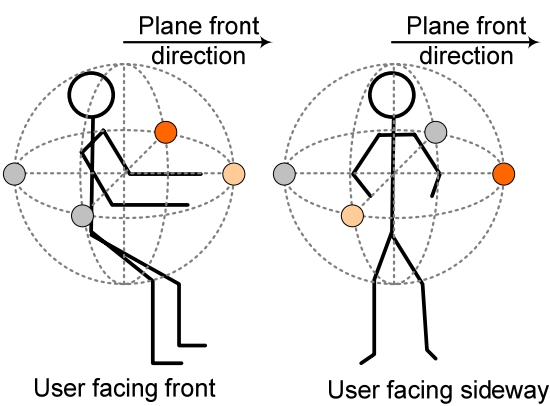

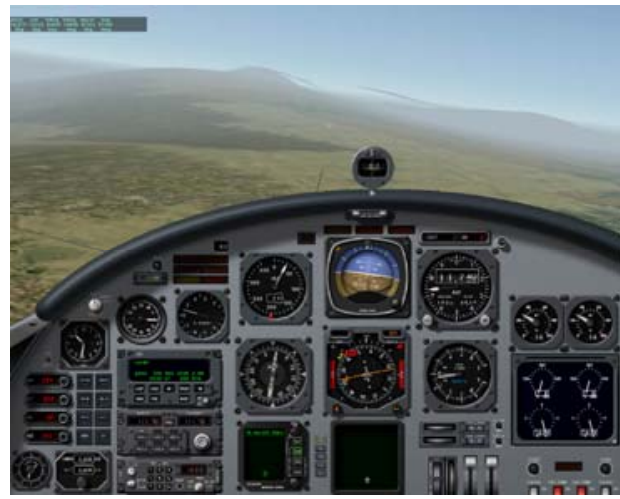

Simple setup using 4 static actuators

One of our concerns is to keep the orientation cue coherent while the pilot is performing necessary movements, such has leaning to pick up objects, feeding time, napping, or reading other information. We have included an orientation sensor which tracks the user's body positioning. We will use four sensors around the user's upper torso dedicated to the roll angle. Keeping the vibration in relation with the orientation of the plane and not the user position will keep coherence of the perception of the roll while the user's moving. Two sensors located on the back of the head and on the belly will keep information of the pitch angle as the precedent configuration. This system corrects the yaw rotation of the pilot upper body but doesn't take in account the pitch and roll.

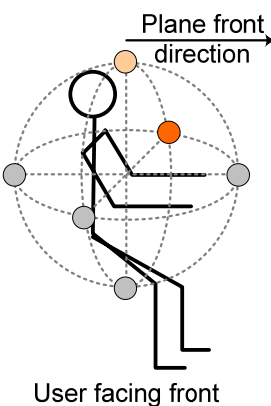

User facing front

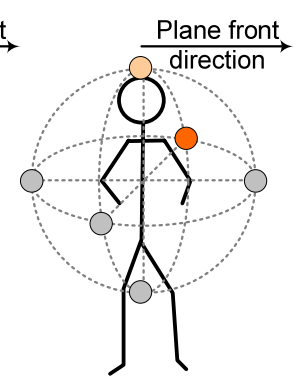

User facing sideway

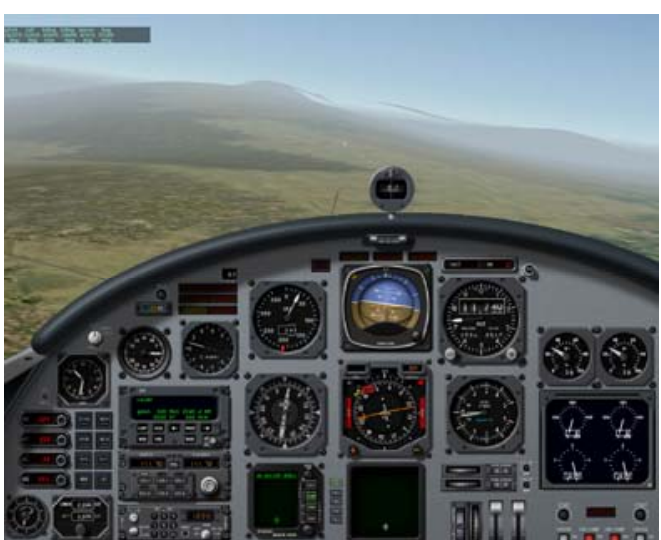




\section{6 actuators setup with user body orientation tracking}

In order to be independent from the user's posture and positioning, we need to insure the coherence of the spatial feedback. The final system includes a set of sensors/actors, actually just 8, distributed onto the user body. Each vibration motor is coupled with a 3D position sensor to track its movements. The roll and pitch angles from the simulator are used to compute the plane's equation corresponding to the current orientation of the aircraft. The plane's origin corresponds to the gravity center of the sensor's positions. The goal is to center the representative feedback in the center of the pilot's own body. This transposition into the user space coordinate is necessary for the cognitive perception of the balance of the plane.

Two different functioning modes have been implemented to pass information about the current positioning of the plane. At first we tried to render directly the attitude of the plane as a 3D "vibrating shape" in space. To do so, we used the distance of each actuator to the vibrating plane. This distance is then correlated to a resulting vibration command for each vibrator. The desired effect creates a continuous vibration plane in space which rotates according to the movement of the plane.

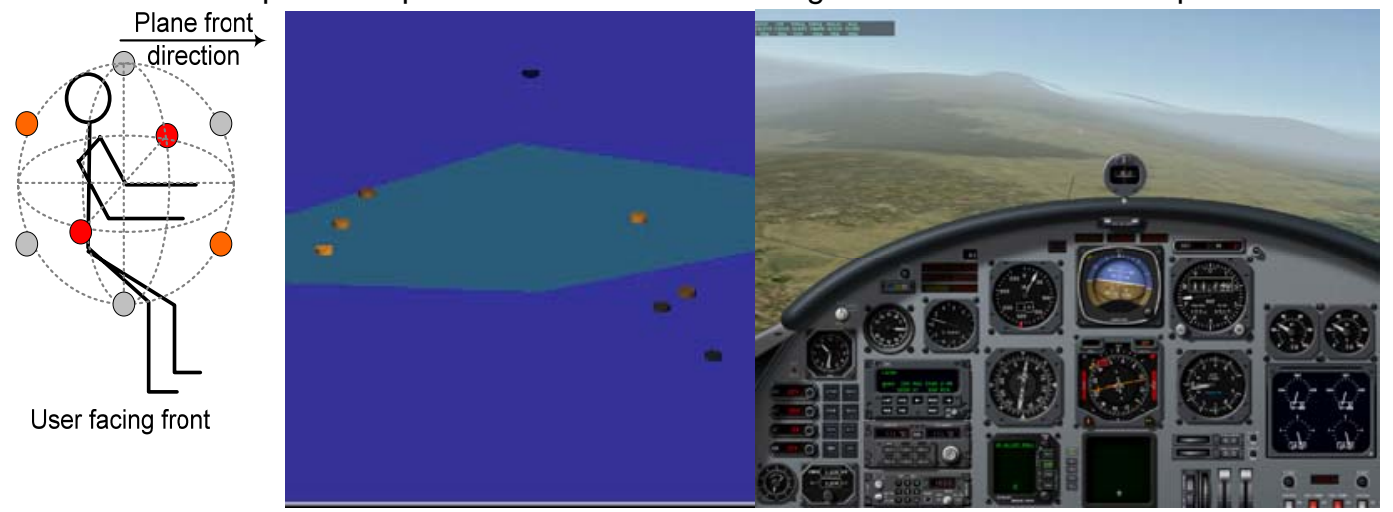

3D shape setup using $\mathrm{N}$ position tracked actuators

The second functioning mode is more applicative. The main disadvantage from the first mode is that the system produces at all time a vibration onto the user's body. It's especially not suitable when the plane is in a flat attitude and there's nothing to report. To avoid a constant vibration feedback we have correlated the vibration to the difference of the plane attitude to a flat position. The position of the vibrator is used to render a maximum vibration in the direction of the difference. For example, if the plane is leaning left, a gradient of vibration is rendered on the vibrators located on the left of the user. The vibration increases in relation with the increment of the roll angle.

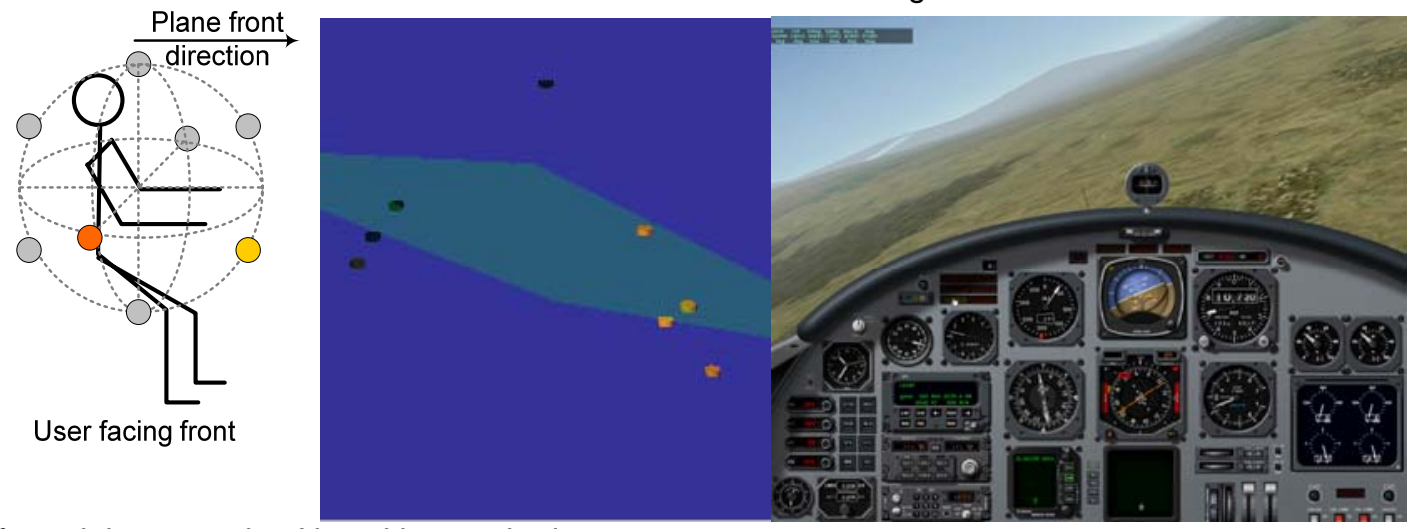

Differential setup using $\mathrm{N}$ position tracked actuators

The information to render onto the vibrator is coded as a 200 ms burst of vibration with variable intensity every second. This technique has been used to not overwhelm the user skin and avoid attenuation of his sensitivity to the stimuli. In order to normalize the rendered vibration according to the variable skin sensitivity of the user, we are using for each vibrator a sensitivity curve issued from individual calibration. This curve is a Spline which is used to adjust the user feedback according to the state of the system. The curve is set using three control points corresponding respectively to the detection threshold, an average excitation and the maximum intensity as presented in the figure below. 


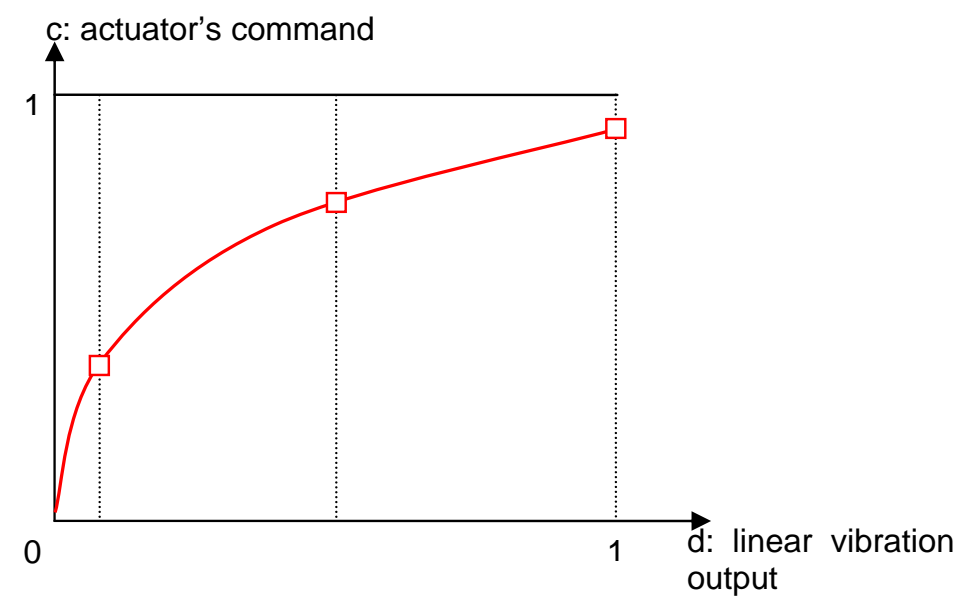

The application purpose is to present the influence of dynamic positioning of the sensor onto the user's body rather than giving a predefined position to each sensor according to the standard position of the limbs that they are fixed upon. The principle is to give feedback which is continuous in the space independent of the pilot's own movement. One of the two main reasons for taking these movements into account are for the purpose of alerting the user when he is performing other movements not directly inherent with piloting, like eating, reading, relaxing or even napping... The other point is to take advantage of the natural leaning while performing a turn. By moving its own gravity center it will also displace the gravity center for the feedback which will follow while keeping the orientation information.

The following part will present the implementation of those principles. It will develop the technical details concerning the flight simulator to recreate the use case situation, the motion capture system used to track the orientation and the position of the user body, the feedback engine which implements the different modes described above and then the embedded vibration feedback which command the actuators.

\section{System architecture}

The system consists of four distinct parts, three of them running on their own computer and communicating with the local network and the last one running on an embedded microcontroller:

- Flight simulator: is running the $\mathrm{X}$ Plane flight simulator $[\mathrm{xx}]$ to give realistic simulation of in flight condition.

- Motion capture: keeps tracks of the position of the actuators on the user body

- Feedback engine: control a 3D interface displaying information about the vibrating system state

- Embedded vibration system: communicates with the feedback engine through Bluetooth communication and control the vibration motors.

\section{Flight simulator}

In order to put the user in piloting condition we use the aircraft simulator, X-Plane, made by Austin Meyer. This software is a commercial application which is used world widely for flight training purpose and certified by the Federal Aviation Administration. It offers a high realistic flight simulation and allows external control on numerous output and input parameters over the simulation.

A standard PC is used to run the simulator and display the image of the internal view of the plane on a video projector. It used a real model of plane used for long flight mission and simulated realistic it's in flight behavior. A simple joystick and thruster are used to pilot the plane to insure to be close to reality. The $X$ plane interface allows us to broadcast over the network various information, like the attitude angles, used in this application. 


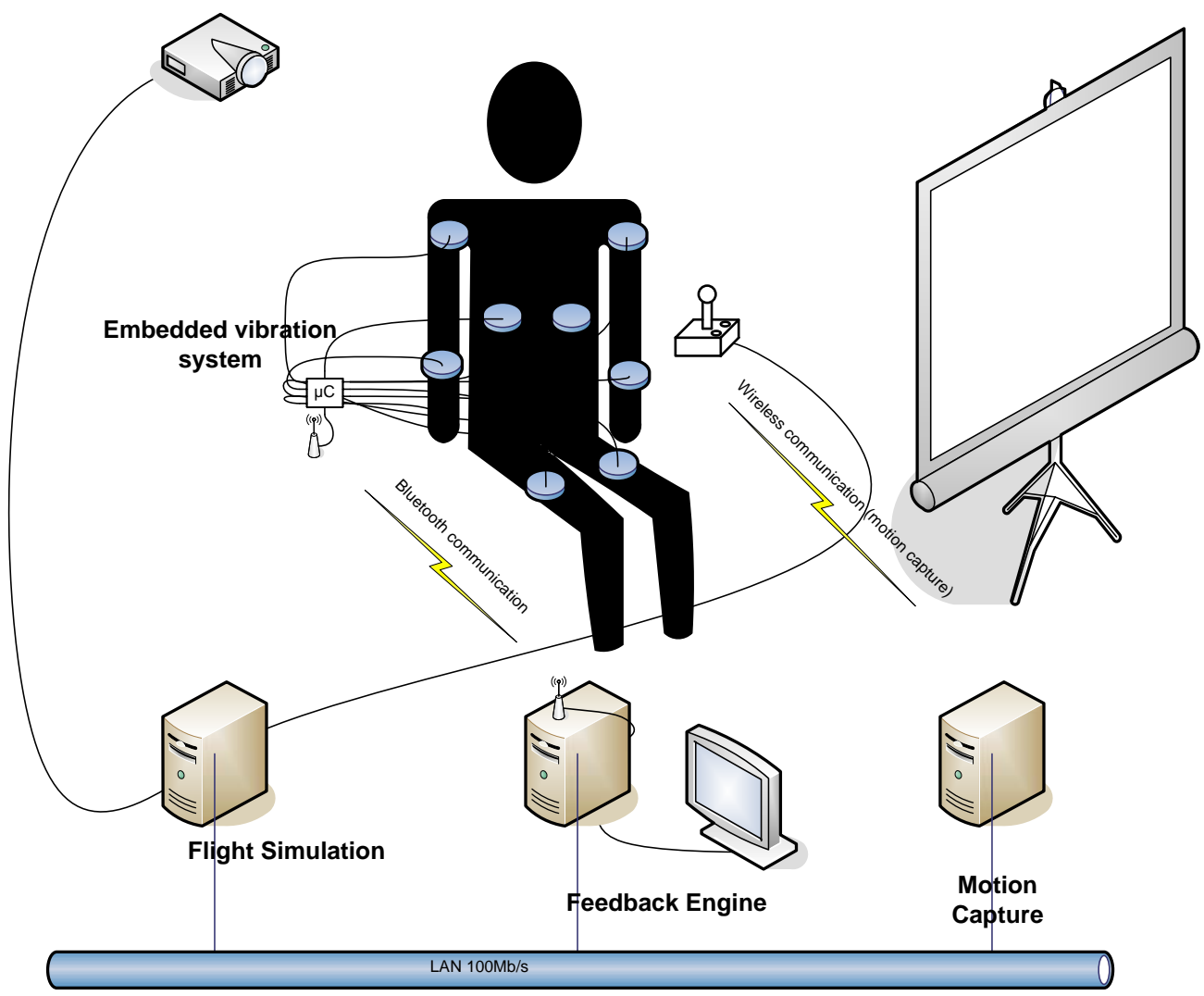

\section{Motion capture system}

In order to track the position of the actuators onto the user body, we are using the magnetic tracking system Motion Star [xxi] from Ascension Technology. Essentially, a source generates a low frequency magnetic field detected by several 3 axis sensors. The trackers provide six degrees of freedom (6DOF) for simultaneously tracking the position and orientation of the receivers (sensors) over a specified range of 3 meters. Motions of the sensors are tracked to accuracies of $0.5^{\circ}$ and $3 \mathrm{~mm}$ at rates up to $144 \mathrm{~Hz}$. The sensors acquisition is done by an embedded acquisition module connected via wireless to the data treatment module. The user is able to move and rotate freely without any constraints. The motion capture system stores the position and orientation information into a shared memory over the network. A library has been developed to access this information in real time from a distant machine. The library creates a local copy of the motion capture buffer data, synchronize it and return a pointer to this buffer.

\section{Embedded Vibration system}

The embedded vibration system is based around an 8 bits micro-controller PIC18F6720 [xxii]. This device recovered the data to output from the feedback engine via its serial port. The micro-controller emulates the Pulse Width Modulation (PWM) outputs which drives the vibration motors. The PWM signal frequency is about $200 \mathrm{~Hz}$ with amplitude of 3.8 volts which correspond to the nominal tension specified by the design of the vibration motors.
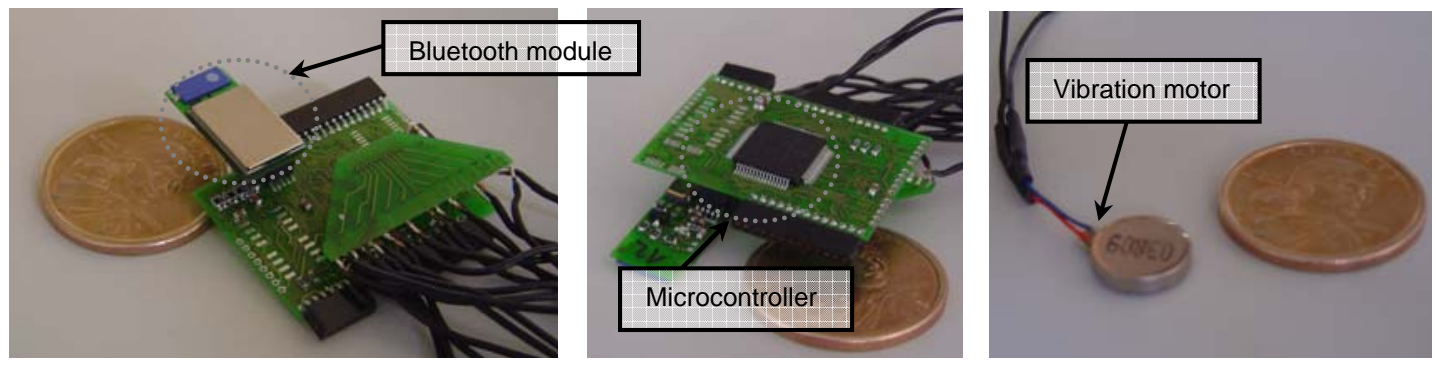
The microcontroller has 8bits architecture without any operating system. The software is based on peripheral interruptions. This device is designed to run at $40 \mathrm{MHz}$ and able to drive 49 inputs and outputs with embedded specialized hardware.

For gathering information form the feedback engine, a Bluetooth module is paired with the remote computer to enable wireless serial communication. The microcontroller received data thru its Universal Serial Asynchronous Receiver Transmitter (USART) hardware. The system generates a hardware interrupt each time a complete byte is received. Then the software recovers the data, stores and organizes it as a received packet which contains information about all the PWM outputs.

The emulation of the PWM output is based on an internal backward counter which can be set and generates an interrupt on reset. The tricks used to emulate different outputs out of a single timer is to computes the different time steps separating each change of the outputs as a whole entity. The software sets the counter to this time steps and updates the values of the outputs with the desired values when the time step is over. In order to provide error proof communication, a minimal protocol packet has been used between the computer and the microcontroller providing a header, length and ending byte codes.
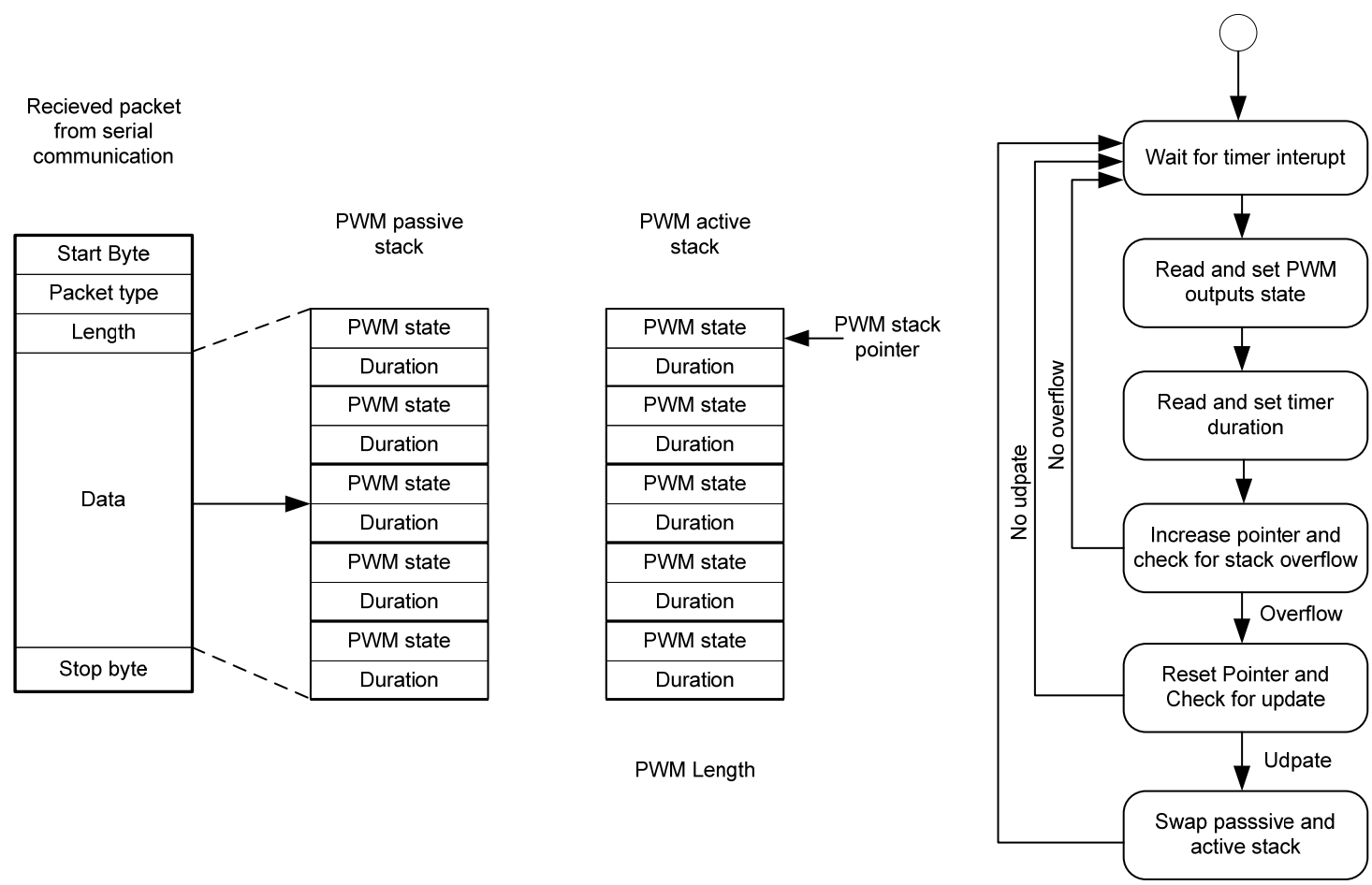

The PWM period of the system is around $5 \mathrm{~ms}$. In order to implement burst of vibration another internal counter with a period of 1 second is used to create software interruptions at 200 milliseconds and on reset. A flag is turned on and off to clear all the PWM outputs between two bursts.

The actuators used to render the tactile information are DC vibration motor issued from the mobile phone technology. For this application, we used the coin motor SAM-A300 which allow easy mounting on the user body. By sending PWM input to this simple brushless DC motor we can adapt its speed and by then the mechanical vibrating energy to transmit through the user skin. These motors are the size of a coin battery for watches which allow better integration into clothing.

\section{Feedback engine}

This is the core of our system. This program gathers the information of the plane behavior and sends back the appropriate feedback to the embedded vibration system. This program recovers the pitch and roll angles of the aircraft from the plane simulator via an UDP connection over the network. Also connected through the shared memory to the motion capture, the program gathers 3D positions of the corresponding actuators.

The interface presents the user a 3D representation of the vibrators in space, a plane representing the current attitude of the aircraft and the rendered vibrations by the color of the actuators. The MVisio [xxiii] graphic engine developed in our lab has been used for fast and easy programming. A text list can also display all the different input, output and parameters values. Configuration of the software is 
done via sliders and text boxes to enter and modify easily the vibration engine parameters. A window can be open for each actuator to setup the control points of the calibration curve.

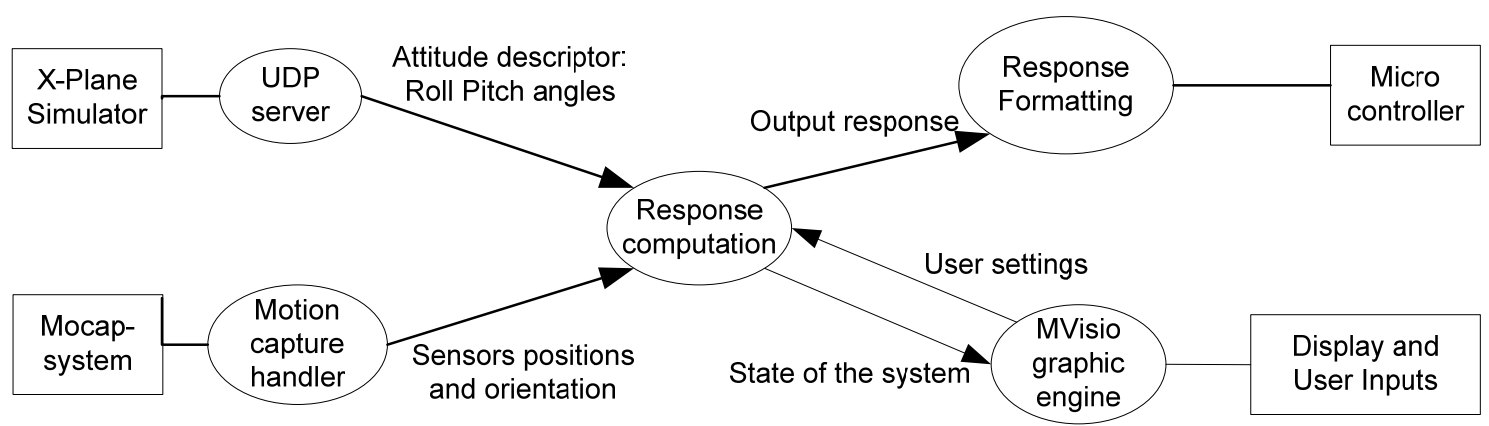

For the purpose of this application the software implements different modes of vibration feedback. Those four modes, described below, are implemented in the "response computation" process and selected by the user.

- The first mode implemented utilizes the sine of the attitude angles as the input value for the 4 actuators located in front / back for the render the pitch, and left / right for the roll. This mode is perfectly static and does not take the value of the motion capture data.

- The next step is to take in account the global orientation of the user using 6 actuators at arbitrary position. The 4 actuators located around the user body render the roll angle taking in account the orientation of the pilot body relative to the plane. The orientation of the user is given by the orientation of one reference sensor from the motion capture system. The roll feedback direction is always given in the one the plane is leaning to, independent of the user's posture in the aircraft

- The last modes use a set of actuators, 8 in our case, each of them tracked with motion capture. The system computes the gravity center of the whole set of actuators and use it at the origin point. The system computes the plane equation from the pitch and roll angles. The rendered vibration is proportional to the distance to the plane of vibration.

- The final setup is based on the same principle. But the resulting vibration from each sensor is calculated from the difference of the plane position to its neutral place at the location of the actuator in the relative space. It means that the system will vibrate in the direction of the plane is leaning independently from the user's position.

.The vibration is calculated as a relative index between 0 and 1 in order to represent the attitude of the plane. For improving the perception, this index is correlated using the calibration curve of the given actuator. A graphical interface allows the user to change the control points of the calibration curve dynamically according to his own sensitivity. In the static localization the user places himself in the standard piloting position and the software record once for all the position of the vibrators. The motion captured here is only a technical tool to measure the position of the actuators. It could have been done by other means just as measuring manually their position and hardcode those positions into the system. In the dynamic geo-localization mode the motion capture keeps track of the vibrators positions at each time step and updates the whole system.

This setup allows proper implementation of the designed approaches for improving piloting conditions during long flights. The next paragraph presents the validation procedure and results to test the usability of such system. By using the different modes of feedback under different use case situations we purposes to determine the added value of the different configurations.

\section{Discussion and results}

The system developed provides us a good platform for testing and validate its usability. In order to place the user as close to reality, we use immersive equipment for the validation. We purpose to display the flight simulator onto a large screen. A video projector has been used to get the display screen covering the whole field of view of the user. The pilot sits with a joystick and a thruster to conform to real piloting position. 


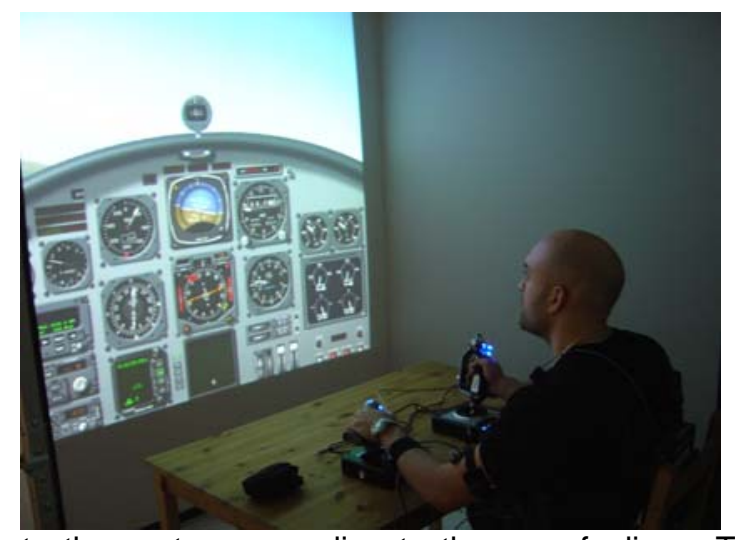

The first step is to calibrate the system according to the user feelings. The user of the system is equipped with a shirt where the vibration motors are fixed in given positions. By placing the control points of the calibration curve, the user indicates the command threshold of each vibration motor according to the provided sensation. Then the user tries to find the uniform maximum of stimulation among all the actuators. For a quick approximation the actuator with the highest threshold is set under the maximum acceptable command. By iterative process, all the other actuators are set to the same perceived level. A middle vibration level can also be defined to insure continuity all along the command variation.

In order to test the usability of the system we measured the user performance onto the simulator under different situations. The situations are selected to provide different visual cues for the plane attitude estimation. The first visual cue is the perception of the horizon which indicates is the natural visual reference during flight. In order to test its influence test has been performed during days and night to simulate absence or presence of this horizontal reference. During day time the user is able to see the horizon line but during night time or when the plane is entering clouds no visual feedback is available. The standard tool to perceive the attitude during night flight is the attitude indicator present in all modern airplane cockpits. To measure the impact of our system, we performed tests by setting on and off the different visual cues and the vibrating device. We added violent bursts of $120 \mathrm{~km} / \mathrm{h}$ lateral wind onto the simulator and measure the time for the pilot to stabilize it on flat attitude. 5 different users have been performing the tests under the different situations. We measures the mean response time and displayed the standard deviation among the different performances.

Influence of the different vibrating feedback according to the available cues

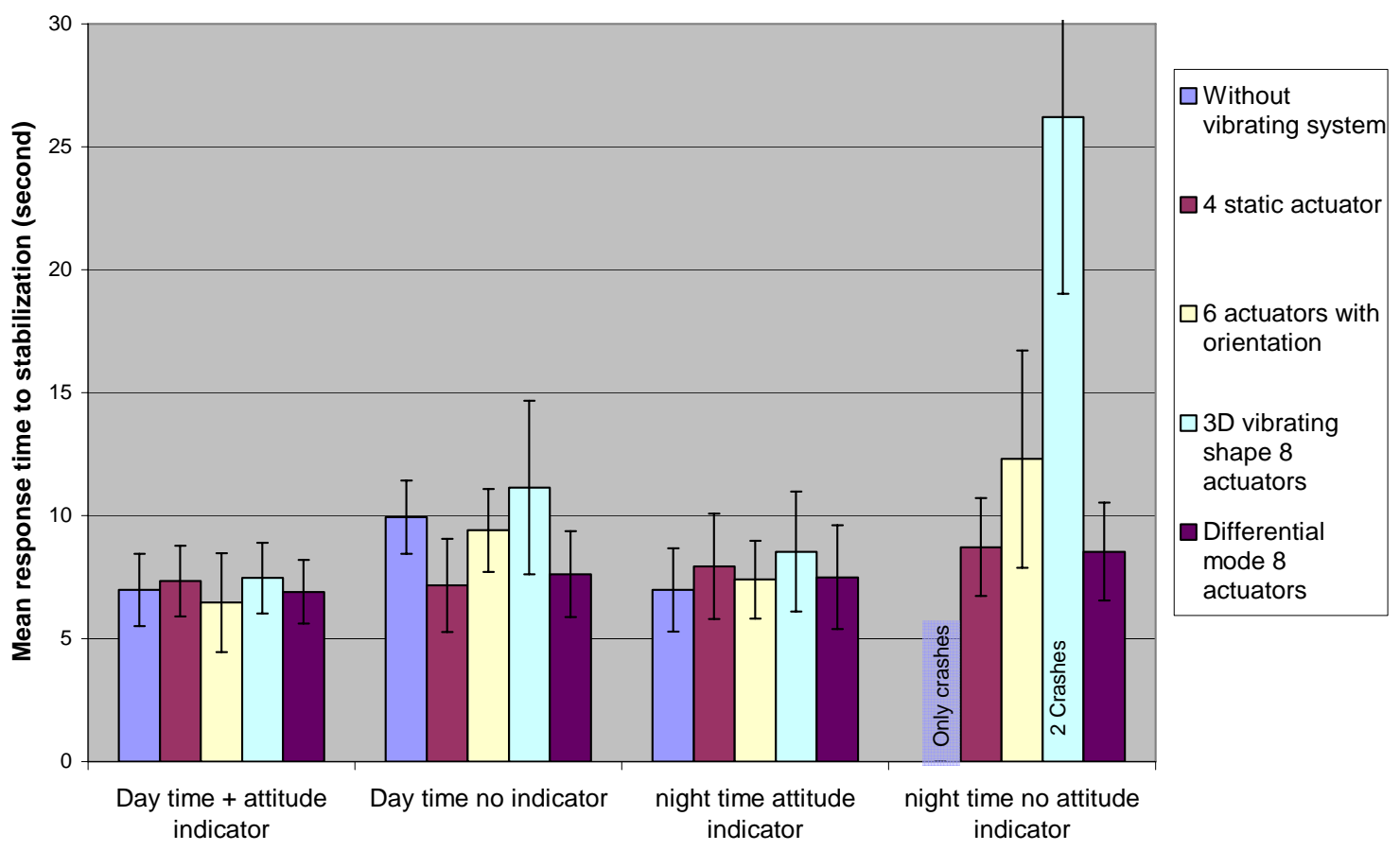


In this graph, we can observe that the user rely mostly on the attitude indicator and the visual cue to take corrective action. When those cues are presented the influence of the vibrating system is limited. The error, presented as the bar, is too high to allow relevant interpretation when the attitude indicator is on. However we distinguish that the 4 actuator setup provided enough information for the pilot to rely on. Similar results can be observed using the 8 actuator setup with dynamic differential mode. This improvement shows the influence of localization of the vibrating feedback. This is especially noticeable on the awaking period where the response time is drastically reduced. The limited number of actuators, eight in the current configuration, is not sufficient to perceive properly the vibrating plane for the absolute mode with a 3D vibrating shape. A break in the presence of the feedback attitude plane is introduced by the absence of vibration where the corresponding user space does not include vibrators. This break can lead to dramatic results when the user only has the vibrating system to rely on. Although we can notice that the static and differential setup, give reliable feedback and compensate the absence of attitude indicator. In this case, the user is even able to take corrective action, only relying on the vibrating system.

In order to determine the pertinence of dynamically localized feedback, we first test the simplest case using four actuators without any kind of localization. This test is used as a reference to test the usability of dynamic localization when the user is moving. For this purpose we make the pilot change his position. Then we measure the effects of the perturbation introduced, according to the different situations. As for the precedent validation, we have done some tests by adding violent bursts of 120 $\mathrm{km} / \mathrm{h}$ lateral wind onto the simulator and measure the time for the pilot to stabilize it. The movements we are asking are voluntarily exaggerated for the validation purpose. A reference is establishing by measuring the pilot response time while sited in the cockpit. The first test represents the pilot performing a daily task onboard requiring access to the back of the cabin. For simulating this, we asked the user to read a text sited and to turn his back to the piloting position. The last test is the simulation of awaking in the cabin. For this purpose we ask the user to lie on a mattress on the floor and to close his eyes and relax for ten minutes Those tests are done to really twist the user position and to see if keeping the vibration as a space constant for a constant attitude does not twist the user interpretation of the feedback.

Influence of user movement and dynamic feedback

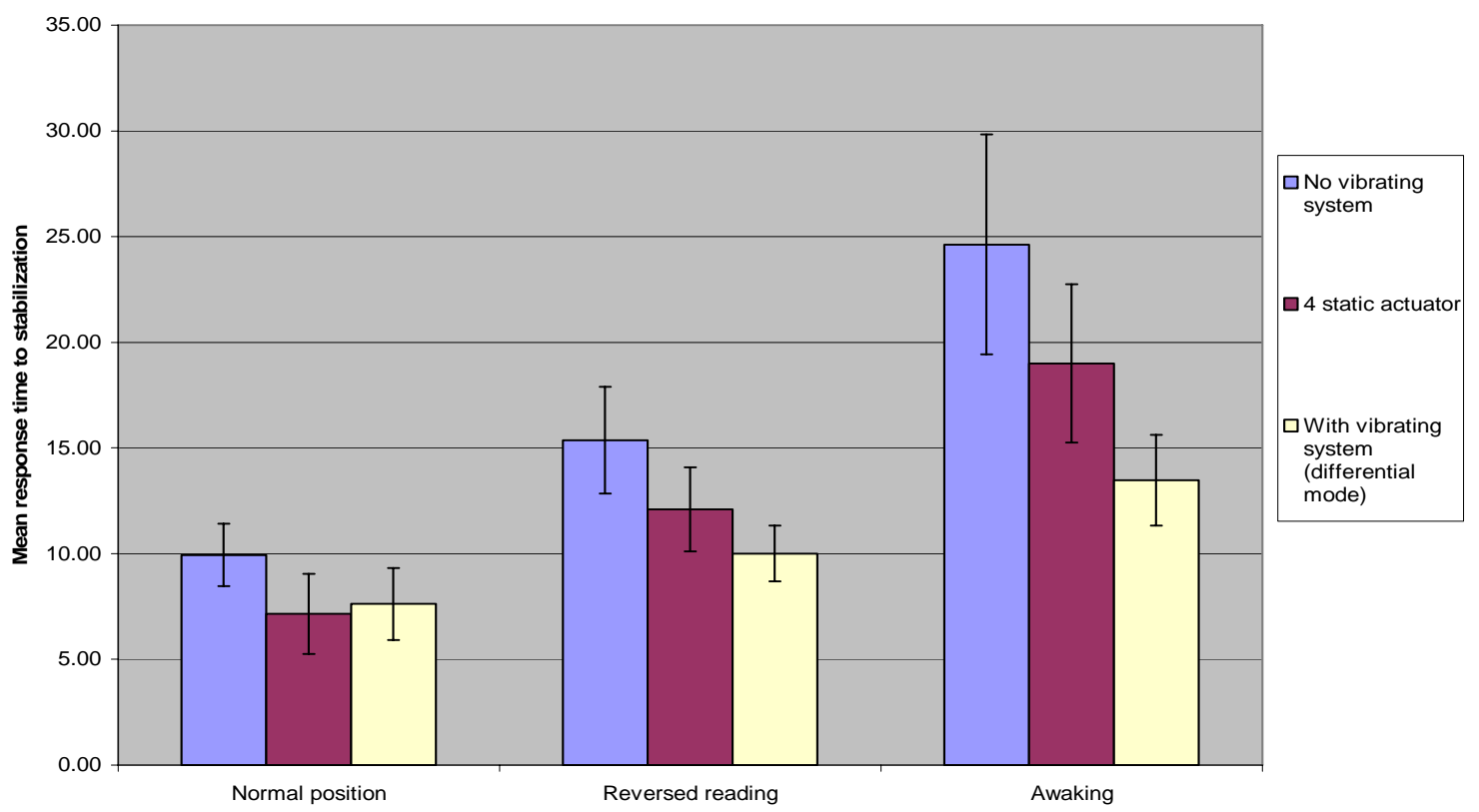

This graph characterizes the use of the dynamic localization system for counter balance the user movement. In fact we can observe that when the user is performing movements in the static mode, he gets false estimation of the attitude while performing other tasks and especially at the awaking phase. This is translated by a slower response time to stabilize the aircraft. The added value of dynamically localized system is noticeable according to lower response during disorientating activities. During the awaking phase the user can regain control of its aircraft faster than with the 4 static actuators setup. Such difference in the response time could be explained by the importance of the very first second 
after the introduction of perturbation. The first commands are crucial to regain control quickly. The dynamical localization gives information about the situation independently from the user body configuration and its associated disorientation during the waking process for example. Those experiments have leaded us to the following conclusion

\section{Conclusion and future works}

In general we can determine the usability of such vibrating system since it gives reduces the stabilization time. The attitude indicator is still necessary due to the lacks of the current configuration. As we can see in the results, the response times are higher without this device. The major advantage of the current vibrating system is coming from the fact that it stimulates the user to catch his attention as well as proving the needed information without the need of reading the flight tools. The system clearly demonstrates the added value of vibrating system while performing other operations involving user movements and awaking alerts.

The wearability of the system needs to be improved in order to insure a proper use in real situations. The current actuators and main embedded units are small enough to be placed in clothing. In order to make the use of this system comfortable enough to be used for several days, it is important to weave the wiring parts directly into the clothing. In order to increase the potential impact validation of this type of system, validation with real pilots is vital. We will perform some tests with some experienced crew members and pilots. During those tests it will be interesting to increase the immersion factor by using a mockup of a real aircraft cockpit or make a virtual simulation using immersive environment available like a stereoscopic cave and/or force feedback devices. In order to improve the current application, we will focus our work on several points. First we need to improve the response time of the system. Since we observe a break in presence using the 6actuators setup and 3D shape modes, the number of actuators has to be increased in order to insure continuity in the spatial vibrating representation. This will allow a better transition of the vibration between actuators. This could only be possible by increasing the frequency of the microcontroller to emulate more PWM output and/or by using a network of embedded microcontrollers. Improvement will also be done on the vibrating feedback itself by improving the vibration engine. More fine tuning needs to be done on the actual available mode to find the optimum feedback. We will also try to develop new vibrating paradigm exploiting the extra sensitivity of the skin to temporal responses. It will be interesting to use induced vibrating waves in space to pass spatial information. This could be done by playing with decays between the different PWM outputs.

Localized vibrating system shows improvement of piloting condition and lowers the reaction time in case of emergency. The localization of the actuators in reference to the user posture into its cockpit allows spatial coherence of the resulting vibrating feedback. It improves the response time to regain control of the aircraft while performing daily activities or when the attention is reduced due to sleep deprivation.

\section{References}

[i] G.P. Taylor, "Warfighter endurance management during continuous flight and ground operation".

[ii] Guerraz M, Poquin D, Ohlmann T., (1998) The role of head-centric spatial reference with a static and kinetic visual disturbance, Percept Psychophys.60(2), pp 287-95.

[iii]S. Durnford, J. Crowley, N. Rosado, J. Harper, S. Deroche, "Spatial disorientation: A survey of U.S. Army helicopter accidents" 1987- 1992

[iv]J. Van Erp, H. Van Veen, C. Janseen, T. dobbins, (2005) "Vibrotactile Waypoint Navigation at Sea and in the Air: two Case study", ACM Transactions on Applied Perception,

[v] Rupert A.H:, McGrath, B.J., Griffin M.(2002).A Tool to Maintain Spatial Orientation and Situation Awareness for Operators of Manned and Unmanned Aerial Vehicles and other Military Motion platforms, RTO HFM Symposium on "spatial Disorientation in Military Vehicles: Causes, Consequences and Cures", 31.1-31.15.

[vi]. van Erp J.B.F (2002), "Guidelines for the use of vibro-tactile displays in Human computer interaction". In Proc. Of Eurohaptics 2002, University of Edinburgh, Scotland, pp 18-22.

[vii] Verrillo R T, (1965) "Temporal summation in vibrotactile sensitivity" Journal of the Acoustical Society of America $37843-846$

[viii] Hahn, J. F. (1966). Vibrotactile adaptation and recovery measured by two methods. Journal of Experimental Psychology, 71, 655-658.

[ix] Craig, J.C. (1972). Difference threshold for intensity of tactile stimuli. Perception \& Psychophysics, $11,150-152$. 
[x] Goff, G.D. (1967). Differential discrimination of frequency of cutaneous mechanical vibration, Journal of Experimental Psychology, 74, 294-299.

[xi] Gescheider, G.A. (1974). Temporal relations in cutaneous stimulation. In: Cutaneous communication systems and devices, edited by F.A. Geldard. Austin, Tex.: The Psychonimic society.

[xii] van Erp J.B.F (2005), Vibrotactile spatial acuity on the torso: effects of location and timing parameters. In Proceedings of the First Joint Eurohaptics Conference and Symposium on Haptic interfaces For Virtual Environment and Teleoperator Systems (Whc'05) - Volume 00, pp 80-85

[xiii] A.P. Hillstrom, K. Shapiro, \& C. Spence. Attentional and perceptual limitations in processing sequentially presented vibrotactile targets. Perception \& psychophysics 64, 2002, pp. 1068-1082.

[xiv] Craig J.C. \& Evans, P.M. (1987). Vibrotactile masking and the persistence of tactual features. Perception \& Psychophysics, $42,309-317$

[xv]Hahn, J. F. (1966). Vibrotactile adaptation and recovery measured by two methods. Journal of Experimental Psychology, 71, 655-658.

[xvi]Gallace A., Tan H. Z., Spence C, (2005) Tactile change detection, In Proceedings of the First Joint Eurohaptics Conference and Symposium on Haptic interfaces For Virtual Environment and Teleoperator Systems (Whc'05) - Volume 00, pp. 12-16

[xvii] Yang U, Jang Y., Kim G.J., (2002) Designing a Vibro-Tactile wear for "Close Range" Interaction for VR-based Motion Training, ICAT 2002

[xviii] Wexler, M., Panerai, F., Lamouret, I., and Droulez, J. (2001). Self-motion and the perception of stationary objects. Nature, 409:85.88.

[xix]van Erp J.B.F and van Veen, H.A.A.C. (2003)"A Multipurpose Tactile Vest for Astronauts in the International Space Station",Proc. Of Eurohaptics 2003, pp 405-408

[xx] A. Meyer, www.x-plane.com

[xxi] Ascention technology Corporation, http://www.ascension-tech.com

[xxii] Microchip, http://www.microchip.com

[xxiii] 3D Mental Vision project, http://vrlab.epfl.ch/ apeternier/mvisio.html 